\title{
An FMCDM approach to purchasing decision-making based on cloud model and prospect theory in e-commerce
}

\author{
Hong-yu Zhang \\ School of Business, Central South University \\ Changsha 410083, China \\ E-mail:hyzhang@csu.edu.cn \\ Rui Zhou \\ School of Business, Central South University \\ Changsha410083, China \\ E-mail: zrconan@163.com \\ Jian-qiang Wang* \\ School of Business, Central South University \\ Changsha 410083, China \\ E-mail:jqwang@csu.edu.cn \\ Xiao-hong Chen \\ School of Business, Central South University \\ Changsha 410083, China \\ E-mail:csums_2005@163.com
}

Received 13 October 2015

Accepted 27 March 2016

\begin{abstract}
This paper presents a fuzzy multi-criteria decision-making (FMCDM) approach based on cloud model and prospect theory. In addition, a reference point selection method is developed according to the evaluations of the potential customer or similar consumers regarding certain items. An example of purchasing decision problems is provided in order to illustrate the applicability of the proposed approach. A comparative analysis of the proposed approach is also performed in order to verify its feasibility.
\end{abstract}

Keywords: Purchasing decision-making, cloud model, prospect theory, reference point.

\section{Introduction}

Purchasing decision problems involve the evaluations of certain products and their alternatives ${ }^{1}$. Both internal and environmental factors affect the choices of customers. Intuitive decision-making allows customers to bypass the conscious steps of product selection ${ }^{2}$. However, oftentimes potential customers are facing so much information, especially in e-commerce, that they find making intuitive purchasing decisions difficult. Such problems impel customers to use specific approaches that facilitate the appropriate selection of

\footnotetext{
* Corresponding author. Tel.: +86 731 88830594; fax: +86 731 88710006. E-mail address: jqwang@csu.edu.cn.
} 
products or their alternatives. Up to now, some studies concerning purchasing frameworks and theories have been conducted. Nicosia ${ }^{3}$ proposed a purchasing decision-making process model, in which it contained four stages: communication, searching, action and feedback. Engel et al. ${ }^{4}$ put forward an Engel-KollatBlackwell (EKB) model, which was a framework of analyzing consumers' behaviors. Besides, some theories and models related to online purchasing decisionmaking problems were put forward ${ }^{5-9}$. However, these studies only provided a guiding framework for studying purchasing decision-making problems, but they hardly offered an approach with specific procedures to help customers make purchasing decisions. To overcome this deficiency, both theory and practicality should be considered when developing an approach to purchasing decisions.

Fuzzy multi-criteria decision-making (FMCDM) approach was introduced in the early $1970 \mathrm{~s}^{10}$ and was developed as a promising decision-making tool for complex problems with multi-criteria and fuzzy information ${ }^{11-20}$. In essence, a purchasing decisionmaking problem can be expressed as an FMCDM problem. In an e-commerce environment, customers make purchasing decisions according to the items' linguistic evaluations from several aspects ${ }^{21,22}$, which are consistent with the multi-criteria of decision-making problems. The evaluations provided by other consumers, or product reviews, are actually the criteria values. Furthermore, decision-making procedures involve the identification of compromises among several complex and conflicting items. Therefore, a purchasing decision-making problem can be tackled by FMCDM approaches.

Due to the complexity of products and the vagueness of human thinking, people used to give evaluations of items with linguistic information ${ }^{23}$. In some ecommerce websites, such as Taobao.com and Tmall.com, consumers are allowed to give evaluations of items under certain criteria after consuming, and these evaluations are often given with specific linguistic descriptors such as terrible, poor, medium, good and excellent, according to the websites. Although there are some methods, such as linguistic computational models based on membership functions ${ }^{24-26}$, linguistic operators ${ }^{27-29}$ and 2-tuple linguistic models ${ }^{30-34}$, that can be used in decision-making problems with linguistic information, these methods have some limitations in solving practical purchasing decision-making problems ${ }^{35-37}$. Cloud model was developed based on probability theory and fuzzy set theory ${ }^{38}$, it allows for the transformation of linguistic terms into three associated numerical characteristics, which overcomes the limitations of aforementioned methods ${ }^{39}$. Recently, cloud models have been widely applied to practical problems with linguistic information ${ }^{40-42}$, including some decision-making problems ${ }^{43,44}$.

In reality, because of the complexity of purchasing decisions and the cognitive limitations of customers, customers often exhibit bounded rationality ${ }^{9}$. Based on the "bounded rationality" principle ${ }^{45}$, prospect theory was developed ${ }^{46}$ and applied into decision-making problems for its capability of describing the decisionmaking behaviors of individuals at risk of loss ${ }^{47}$. In prospect theory, items are compared according to their prospect values, which reflect individuals' attitudes toward items under risk ${ }^{46}$. Due to online purchasing decisions containing numerous uncertainties and risks, customers make comparison among alternative products to reduce loss and select "most satisfied" items rather than "highest value" items. In order to calculate prospect values of items, reference point, which is considered as the equilibrium point of individual's psychological expectation, must be selected because different reference points result in different outcomes ${ }^{46}$. In some applications of prospect theory, five variables, including the zero point, expected value point, median value of an ordered sequence, worst point, and optimal point, are usually regarded as reference points ${ }^{48}$. In practical purchasing decision problems, different customers may have completely different expectations to the same item, let alone different items. Thus, reference point selection principles must be flexible, rather than fixed, in order to meet the needs of different customers.

In this study, an FMCDM approach to e-commerce purchasing decision problems was developed based on cloud model and prospect theory. Linguistic evaluations were processed using the cloud model and prospect theory was used to compensate for the bounded rationality of customers. In addition, reference point selection principles were investigated in order to improve the validity of the proposed approach.

The remainder of this paper is organized as follows. In Section 2, some basic concepts and definitions related to cloud model, prospect theory, and cloud 
prospect values are introduced briefly. In Section 3, the selection principles of reference points are discussed. An FMCDM approach based on cloud model and prospect theory is proposed in Section 4. In Section 5, an illustrative example is provided, and the proposed FMCDM approach is compared to several other methods in order to confirm its validity and applicability. In Section 6, the conclusions of this paper and information concerning future studies are presented.

\section{Preliminaries}

In this section, we introduce some basic concepts and definitions related to cloud model, prospect theory, and cloud prospect values that will be used throughout this paper.

\subsection{Cloud model}

Definition $1^{39}$. Let $U=\{x\}$ be the universe of discourse, and $T$ is a linguistic term associated with $U$. The degree of membership $C_{T}(x)$ of $x$ in $U$ to the linguistic term $T$ is a random number with a stable tendency. $C_{T}(x)$ takes the values in [0,1]. A normal compatibility cloud is a mapping from the universe of discourse $U$ to the unit interval $[0,1]$, and every $x$ with a degree of membership $C_{T}(x)$ is defined as a cloud drop, such that

$$
\begin{aligned}
C_{T}(x): U & \rightarrow[0,1], \\
\forall x \in U, \quad x & \rightarrow C_{T}(x) .
\end{aligned}
$$

The qualitative meaning of a linguistic term can be expressed by a normal compatibility cloud with three digital characteristics, and a cloud can be described as $Y(E x, E n, H e)$, where the expected value $E x$ is the most representative and typical value of $T$. The entropy $E n$, which describes the fuzziness of the linguistic term, reflects the acceptable range of that linguistic term in the universe of discourse. The hyper entropy $\mathrm{He}$, the second-order entropy of the entropy En, reflects the dispersion and randomness of the degree of membership $C_{T}(x)$.

Definition $2{ }^{49}$. Let $Y_{1}=\left(E x_{1}, E n_{1}, H e_{1}\right)$ and $Y_{2}=\left(E x_{2}, E n_{2}, H e_{2}\right)$ be two arbitrary normal clouds.

The distance $d\left(Y_{1}, Y_{2}\right)$ between $Y_{1}$ and $Y_{2}$ is defined as

$$
d\left(Y_{1}, Y_{2}\right)=\sqrt{\frac{1}{2}\left(\left|E x_{1}-E x_{2}\right|^{2}+\left|E n_{1}-E n_{2}\right|^{2}+\left|H e_{1}-H e_{2}\right|^{2}\right)}
$$

Definition $3{ }^{44}$. Let $Y_{1}=\left(E x_{1}, E n_{1}, H e_{1}\right)$ and $Y_{2}=\left(E x_{2}, E n_{2}, H e_{2}\right)$ be any two normal clouds in the universe of discourse and $Y^{*}=\left(\max _{i} E x_{i}, \min _{i} E n_{i}\right.$, $\left.\min _{i} H e_{i}\right)$ be the positive ideal cloud. When $i=1,2$, the degree of possibility of $Y_{1} \geq Y_{2}$ denoted as $P\left(Y_{1} \geq Y_{2}\right)$ is defined as

$$
P\left(Y_{1} \geq Y_{2}\right)=1-\frac{d\left(Y^{*}, Y_{1}\right)}{d\left(Y^{*}, Y_{1}\right)+d\left(Y^{*}, Y_{2}\right)},
$$

where $d\left(Y^{*}, Y_{1}\right)$ represents the distance between $Y_{1}$ and $Y^{*}$, and $d\left(Y^{*}, Y_{2}\right)$ represents the distances between $Y_{2}$ and $Y^{*}$. If $P\left(Y_{1} \geq Y_{2}\right) \geq 0.5$, then $Y_{1} \geq Y_{2}$; otherwise, $Y_{1}<Y_{2}$.

Definition $4{ }^{50}$. Assume that $\Omega$ is the set of all clouds and $Y_{i}=\left(E x_{i}, E n_{i}, H e_{i}\right)(i=1,2, \cdots, n)$ is a subset of $\Omega$. The mapping CWAA: $\Omega^{n} \rightarrow \Omega$ is defined as the cloudweighted arithmetic averaging (CWAA) operator, such that

$$
\begin{aligned}
& C W A A_{\omega}\left(Y_{1}, Y_{2}, \cdots, Y_{n}\right)=\sum_{i=1}^{n} \omega_{i} Y_{i}=\left(\sum_{i=1}^{n} \omega_{i} E x_{i}, \sqrt{\sum_{i=1}^{n} \omega_{i} E n_{i}^{2}},\right. \\
& \left.\sqrt{\sum_{i=1}^{n} \omega_{i} H e_{i}^{2}}\right) .
\end{aligned}
$$

In Equation (3), $\omega=\left(\omega_{1}, \omega_{2} \cdots, \omega_{n}\right)$ represents the associated weight vector of $Y_{i}\left(E x_{i}, E n_{i}, H e_{i}\right)$ $(i=1,2, \cdots, n), \omega_{i} \in[0,1](i=1,2 \cdots, n)$, and $\sum_{i=1}^{n} \omega_{i}=1$. In particular, when $\omega=\left(\frac{1}{n}, \frac{1}{n}, \cdots \frac{1}{n}\right)$, the CWAA operator will reduce to the cloud arithmetic average (CAA) operator, defined as

$$
\begin{aligned}
& C A A_{\omega}\left(Y_{1}, Y_{2}, \cdots Y_{n}\right)=\sum_{i=1}^{n} \frac{1}{n} Y_{i}=\left(\sum_{i=1}^{n} \frac{1}{n} E x_{i}, \sqrt{\sum_{i=1}^{n} \frac{1}{n} E n_{i}^{2}},\right. \\
& \left.\sqrt{\sum_{i=1}^{n} \frac{1}{n} H e_{i}^{2}}\right) .
\end{aligned}
$$

\subsection{Prospect values and cloud prospect values}

Prospect values are codetermined by the value function and probability weight function ${ }^{46}$, which can be expressed as 


$$
V=\sum_{i=1}^{n}\left(\pi\left(p_{i}\right) v\left(\Delta x_{i}\right)\right),
$$

where $V$ is the prospect value, $p_{i}$ represents the probability of the $i^{\text {th }}$ condition, and $\pi\left(p_{i}\right)$ is the probability weight function of the probability assessment, which reflects the attitude of a decisionmaker regarding risk. In addition, $v\left(\Delta x_{i}\right)$ is the value function, which is based on the subjective feelings of a decision-maker regarding an item; and $\Delta x_{i}$ represents the degree of deviation of a particular value from the reference point. The outcome is identified as a gain if the degree of deviation is a positive value; otherwise, the outcome is identified as a loss.

Definition $5^{44}$. Assume that $Y_{1}\left(E x_{1}, E n_{1}, H e_{1}\right)$ and $Y_{2}\left(E x_{2}, E n_{2}, H e_{2}\right)$ are any two clouds in the universe of discourse, and let $Y_{2}$ be the reference point. The cloud prospect value of $Y_{1}$ can be calculated as

$$
V\left(Y_{1}\right)=v\left(Y_{1}\right) \pi\left(p_{1}\right)
$$

The cloud value function $v\left(Y_{1}\right)$ shown in Equation (6) can be expressed as

$$
v\left(Y_{1}\right)=\left\{\begin{array}{c}
\left(d\left(Y_{1}, Y_{2}\right)\right)^{\alpha}, Y_{1} \geq Y_{2}, \\
-\lambda\left(d\left(Y_{1}, Y_{2}\right)\right)^{\beta}, Y_{1}<Y_{2},
\end{array}\right.
$$

where $d\left(Y_{1}, Y_{2}\right)$ represents the distance between $Y_{1}$ and $Y_{2}$. It is identified as a gain when the value of $Y_{1}$ is greater than that of $Y_{2}$. In the same way, it is a loss when the value of $Y_{1}$ is smaller than that of $Y_{2}$. The values of $Y_{1}$ and $Y_{2}$ can be compared using the degree of possibility mentioned in Definition 3. In addition, $\alpha$ and $\beta$ represent the degree of concavity or convexity of the value function within the regions of gain and loss, respectively and $\alpha, \beta \in(0,1)$. Higher values of $\alpha$ and $\beta$ indicate that the decision-maker is more likely to seek risks. $\lambda$ is used to represent the degree of severe feelings toward loss. Usually, $\lambda>1$, which indicates aversion to loss of individuals.

The cloud probability weight function $\pi\left(p_{1}\right)$ in Equation (6) can be expressed as

$$
\pi\left(p_{1}\right)=\left\{\begin{array}{l}
\frac{p_{1}^{\gamma}}{\left(p_{1}^{\gamma}+\left(1-p_{1}\right)^{\gamma}\right)^{1 / \gamma}}, Y_{1} \geq Y_{2}, \\
\frac{p_{1}^{\delta}}{\left(p_{1}^{\delta}+\left(1-p_{1}\right)^{\delta}\right)^{1 / \delta}}, Y_{1}<Y_{2},
\end{array}\right.
$$

where $p_{1}$ represents the probability of $Y_{1}, \gamma$ and $\delta$ represent the decision-maker's attitude towards riskgain and risk-loss, respectively.

\section{Reference point selection principles}

Selecting a proper reference point for each decisionmaker is necessary in order to effectively construct a purchasing decision-making approach based on prospect theory. However, asking each decision-maker to provide a reference point is unpractical. A reference point can be inferred based on the item preferences of a customer. The item preferences of a customer can be identified via two methods. One is the method in which the preferences of a decision-maker can be directly inferred by collecting and analyzing previous decision-making experiences. The other is inferring the preferences of a decision-maker based on the decision-making experiences of decision-makers with similar preferences. Thus, reference points can be selected based on the previous decision-making experiences of a decision-maker or similar decision-makers.

A reference point represents the equilibrium point of decision-maker's psychological expectation for a particular item. The reference point of a decision-maker can be identified based on his or her previous decisionmaking experiences to determine whether that decisionmaker will experience a gain or loss if he or she purchases particular item.

However, if a decision-maker does not have previous decision-making experiences, the customer's reference point of that item will be inferred based on the past decision-making experiences of other similar decisionmakers.

In this section, two algorithms are put forward as the reference point selection principles of a particular item in a specific category in order to find proper reference points for purchasing decision-makers. Algorithm 1 is suitable for the situations in which decision-maker have previous evaluations of the items in a specific category; Algorithm 2 is available when the decision-maker does not have previous evaluations.

Algorithm 1. Reference point selection based on
previous evaluations.
Input: Decision-maker's previous evaluations of the
items in a specific category.
Output: Decision-maker's reference point for this type of item under each criterion. 
Step 1: Collect the decision-maker's previous evaluations of the items in a specific category, as shown in Table 1.

Table 1 Linguistic evaluations of item criteria

\begin{tabular}{ccccccc} 
Items & $c_{1}$ & $c_{2}$ & $\cdots$ & $c_{j}$ & $\cdots$ & $c_{n}$ \\
\hline$a_{1}$ & $x_{11}^{R}$ & $x_{12}^{R}$ & $\cdots$ & $x_{1 j}^{R}$ & $\cdots$ & $x_{1 n}^{R}$ \\
$a_{2}$ & $x_{21}^{R}$ & $x_{22}^{R}$ & $\cdots$ & $x_{2 j}^{R}$ & $\cdots$ & $x_{2 n}^{R}$ \\
$\vdots$ & $\vdots$ & $\vdots$ & $\ddots$ & $\vdots$ & $\ddots$ & $\vdots$ \\
$a_{i}$ & $x_{i 1}^{R}$ & $x_{i 2}^{R}$ & $\cdots$ & $x_{i j}^{R}$ & $\cdots$ & $x_{i n}^{R}$ \\
$\vdots$ & $\vdots$ & $\vdots$ & $\ddots$ & $\vdots$ & $\ddots$ & $\vdots$ \\
$a_{m}$ & $x_{m 1}^{R}$ & $x_{m 2}^{R}$ & $\cdots$ & $x_{m j}^{R}$ & $\cdots$ & $x_{m n}^{R}$ \\
\hline & & & & & &
\end{tabular}

In an e-commerce environment, customers usually do not buy the same one for several times, but they often buy various items, and they are allowed to give evaluations of items after consuming. These evaluations given by the customers are considered as the previous evaluations. In Table $1, a_{i}$ denotes the $i^{\text {th }}$ item, $c_{j}$ denotes the $j^{\text {th }}$ criterion, and $x_{i j}^{R}$ denotes the decision-maker's previous evaluation of the $i^{\text {th }}$ item under the $j^{\text {th }}$ criterion, expressed by a linguistic term. The superscript $R$ means "reference point". We use this superscript to distinguish the evaluations that are used for finding reference points from those that are used for making purchasing decisions. This step can be problematic in that there may be no evaluations of an item under a certain criterion. In this paper, the evaluation is assumed to be the mode value of the customer's previous evaluations of the items in the category under specific criterion.

Step 2: Transform the linguistic evaluations into clouds.

Backward cloud generators (BCGs) and golden sections (GSs) are the approaches most frequently used to generate clouds ${ }^{51}$. Most decision-making approaches based on cloud model use GSs to generate clouds for simplicity. However, due to limitations of the applied mathematical calculation methodology, the clouds generated by GSs cannot accurately describe linguistic terms. In contrast, BCGs utilize statistical information to generate clouds, the clouds generated by BCGs describe linguistic terms more accurately than those generated by GSs ${ }^{51}$.
In this step, linguistic evaluations can be aggregated and transformed into clouds using BCGs ${ }^{52-54}$. The results could be shown as $A=\left\{Y_{1}^{R}, Y_{2}^{R}, \cdots, Y_{j}^{R}, \cdots, Y_{n}^{R}\right\}$, where $A$ denotes the category of the item, and $Y_{j}^{R}$ denotes the cloud evaluation aggregation result obtained under the $j^{\text {th }}$ criterion.

Step 3: Use the aggregation result as the reference point.

Use $Y_{j}^{R}$ as the reference point of a decision-maker for item category $A$ under the $j^{\text {th }}$ criterion.

Algorithm 2. Reference point selection based on the previous evaluations of other decision-makers.

Input: Similar decision-makers' evaluations of the items in a specific category.

Output: Decision-maker's reference point of this type of item under each criterion.

Step 1: Collect the evaluations of other similar decision-makers, and show them as Table 2.

Table 2 Linguistic evaluations of item criteria provided by other similar decision-makers

\begin{tabular}{|c|c|c|c|c|c|c|c|}
\hline $\begin{array}{c}\text { Decision- } \\
\text { makers }\end{array}$ & Items & $c_{1}$ & $c_{2}$ & $\cdots$ & $c_{j}$ & $\cdots$ & $c_{n}$ \\
\hline \multirow{5}{*}{$u_{1}$} & $a_{1}^{1}$ & $x_{111}^{R}$ & $x_{112}^{R}$ & $\ldots$ & $x_{11 j}^{R}$ & $\ldots$ & $x_{11 n}^{R}$ \\
\hline & $a_{2}^{1}$ & $x_{121}^{R}$ & $x_{122}^{R}$ & $\ldots$ & $x_{12 j}^{R}$ & $\ldots$ & $x_{12 n}^{R}$ \\
\hline & $\vdots$ & $\vdots$ & $\vdots$ & $\ddots$ & $\vdots$ & $\ddots$ & $\vdots$ \\
\hline & $a_{m^{\prime}}^{1}$ & $x_{1 m^{\prime} 1}^{R}$ & $x_{1 m^{\prime} 2}^{R}$ & $\ldots$ & $x_{1 m^{\prime} j}^{R}$ & $\ldots$ & $x_{1 m^{\prime} n}^{R}$ \\
\hline & $a_{1}^{2}$ & $x_{111}^{R}$ & $x_{112}^{R}$ & $\ldots$ & $x_{11 j}^{R}$ & $\ldots$ & $x_{11 n}^{R}$ \\
\hline \multirow{3}{*}{$u_{2}$} & $a_{2}^{2}$ & $x_{221}^{R}$ & $x_{222}^{R}$ & $\cdots$ & $x_{22 j}^{R}$ & $\ldots$ & $x_{22 n}^{R}$ \\
\hline & $\vdots$ & $\vdots$ & $\vdots$ & $\ddots$. & $\vdots$ & $\ddots$ & $\vdots$ \\
\hline & $a_{m}^{2}$ & $x_{2 m^{\prime} 1}^{R}$ & $x_{2 m^{\prime} 2}^{R}$ & $\ldots$ & $x_{2 m^{\prime} j}^{R}$ & $\cdots$ & $x_{2 m^{\prime} n}^{R}$ \\
\hline \multirow[t]{3}{*}{$\vdots$} & $\vdots$ & $\vdots$ & $\vdots$ & $\ddots$ & $\vdots$ & $\ddots$ & $\vdots$ \\
\hline & $a_{1}^{i}$ & $x_{i 11}^{R}$ & $x_{i 12}^{R}$ & $\ldots$ & $x_{i 1 j}^{R}$ & $\cdots$ & $x_{i 1 n}^{R}$ \\
\hline & $a_{2}^{i}$ & $x_{i 21}^{R}$ & $x_{i 22}^{R}$ & $\ldots$ & $x_{i 2 j}^{R}$ & $\cdots$ & $x_{i 2 n}^{R}$ \\
\hline \multirow{4}{*}{$u_{i}$} & $\vdots$ & $\vdots$ & $\vdots$ & $\ddots$ & $\vdots$ & $\ddots$ & $\vdots$ \\
\hline & $a_{k}^{i}$ & $x_{i k 1}^{R}$ & $x_{i k 2}^{R}$ & $\ldots$ & $x_{i k j}^{R}$ & $\ldots$ & $x_{i k n}^{R}$ \\
\hline & $\vdots$ & $\vdots$ & $\vdots$ & $\ddots$ & $\vdots$ & $\ddots$ & $\vdots$ \\
\hline & $a_{m^{\prime}}^{i}$ & $x_{i m^{\prime} 1}^{R}$ & $x_{i m^{\prime} 2}^{R}$ & $\ldots$ & $x_{i m^{\prime} j}^{R}$ & $\ldots$ & $x_{i m^{\prime} n}^{R}$ \\
\hline \multirow[t]{2}{*}{$\vdots$} & $\vdots$ & $\vdots$ & $\vdots$ & $\ddots$ & $\vdots$ & $\ddots$ & $\vdots$ \\
\hline & $a_{1}^{m}$ & $x_{m 11}^{R}$ & $x_{m 12}^{R}$ & $\ldots$ & $x_{m 1 j}^{R}$ & $\cdots$ & $x_{m 1 n}^{R}$ \\
\hline \multirow{3}{*}{$u_{m}$} & $a_{2}^{m}$ & $x_{m 21}^{R}$ & $x_{m 22}^{R}$ & $\cdots$ & $x_{m 2 j}^{R}$ & $\cdots$ & $x_{m 2 n}^{R}$ \\
\hline & $\vdots$ & $\vdots$ & $\vdots$ & $\ddots$. & & $\ddots$ & $\vdots$ \\
\hline & $a_{m^{\prime}}^{m}$ & $x_{m m^{\prime} 1}^{R}$ & $x_{m m^{\prime} 2}^{R}$ & $\ldots$ & $x_{m m^{\prime} j}^{R}$ & $\ldots$ & $x_{m m^{\prime} n}^{R}$ \\
\hline
\end{tabular}


In this step, all the similar decision-makers of the potential customer, who have purchased the items of the category and offered evaluations, are selected, and the degree of similarity between a potential customer and one of his similar customers can be obtained by using the similarity measurement ${ }^{55-58}$ and the clustering technology ${ }^{59-61}$.

In Table 2, $u_{i}$ denotes the $i^{\text {th }}$ similar decision-maker, $c_{j}$ denotes the $j^{\text {th }}$ criterion, $a_{k}^{i}$ denotes the $k^{\text {th }}$ item of the $i^{\text {th }}$ decision-maker, and $x_{i k j}^{R}$ denotes the $i^{\text {th }}$ similar decisionmaker's evaluation of the $k^{\text {th }}$ item under the $j^{\text {th }}$ criterion, expressed as a linguistic term. The superscript $R$ is utilized to distinguish the evaluations for finding reference points from those for making purchasing decisions.

Step 2: Transform the linguistic evaluations into clouds.

Using BCGs, linguistic evaluations can be aggregated and transformed into clouds, as displayed in Table 3.

Table 3 Cloud evaluations of item criteria provided by other similar decision-makers

\begin{tabular}{ccccccc}
\hline Decision-makers & $c_{1}$ & $c_{2}$ & $\cdots$ & $c_{j}$ & $\cdots$ & $c_{n}$ \\
\hline$u_{1}$ & $Y_{11}^{R}$ & $Y_{12}^{R}$ & $\cdots$ & $Y_{1 j}^{R}$ & $\cdots$ & $Y_{1 n}^{R}$ \\
$u_{2}$ & $Y_{21}^{R}$ & $Y_{22}^{R}$ & $\cdots$ & $Y_{2 j}^{R}$ & $\cdots$ & $Y_{2 n}^{R}$ \\
$\vdots$ & $\vdots$ & $\vdots$ & $\ddots$ & $\vdots$ & $\ddots$ & $\vdots$ \\
$u_{i}$ & $Y_{i 1}^{R}$ & $Y_{i 2}^{R}$ & $\cdots$ & $Y_{i j}^{R}$ & $\cdots$ & $Y_{i n}^{R}$ \\
$\vdots$ & $\vdots$ & $\vdots$ & $\ddots$ & $\vdots$ & $\ddots$ & $\vdots$ \\
$u_{m}$ & $Y_{m 1}^{R}$ & $Y_{m 2}^{R}$ & $\cdots$ & $Y_{m j}^{R}$ & $\cdots$ & $Y_{m n}^{R}$ \\
\hline
\end{tabular}

In Table $3, u_{i}$ denotes the $i^{\text {th }}$ similar decision-maker, $c_{j}$ denotes the $j^{\text {th }}$ criterion, and $Y_{i j}^{R}$ denotes the $i^{\text {th }}$ similar customer's cloud evaluations of item category $A$ under the $j^{\text {th }}$ criterion.

Step 3: Aggregate the cloud evaluations under each criterion.

The cloud evaluations of item category $A$ provided by other similar decision-makers can be aggregated using the CWAA operator in Definition 4 , and the results could be shown as $A=\left\{Y_{1}^{R}, Y_{2}^{R}, \cdots, Y_{j}^{R}, \cdots, Y_{n}^{R}\right\}$, where $A$ denotes the category of the item, and $Y_{j}^{R}$ denotes the cloud evaluation aggregation result under the $j^{\text {th }}$ criterion and $\quad Y_{j}^{R}=\sum_{i=1}^{m} \omega_{i j} Y_{i j}^{R}=\left(\sum_{i=1}^{m} \omega_{i j} E x_{i j}^{R}\right.$,

$\left.\sqrt{\sum_{i=1}^{m} \omega_{i j}\left(E n_{i j}^{R}\right)^{2}}, \sqrt{\sum_{i=1}^{m} \omega_{i j}\left(H e_{i j}^{R}\right)^{2}}\right)$. In this paper, we assume all similar decision-makers have the same weight for the simplicity of calculation, thus the CWAA operator is reduced to $\mathrm{CAA}$, and $Y_{j}^{R}=$ $\sum_{i=1}^{m} \frac{1}{m} Y_{i j}^{R}=\left(\sum_{i=1}^{m} \frac{1}{m} E x_{i j}^{R}, \sqrt{\sum_{i=1}^{m} \frac{1}{m}\left(E n_{i j}^{R}\right)^{2}}, \sqrt{\sum_{i=1}^{m} \frac{1}{m}\left(H e_{i j}^{R}\right)^{2}}\right)$.

Step 4: Use the aggregation result as the reference point.

Use $Y_{j}^{R}$ as the reference point of the decision-maker for item category $A$ under the $j^{\text {th }}$ criterion.

\section{FMCDM approach based on cloud model and prospect theory}

In this section, an FMCDM approach based on cloud model and prospect theory, in which the reference point selection principles proposed in Section 3 are applied, is developed.

Let $M=\{1,2, \cdots, m\} \quad$ and $\quad N=\{1,2, \cdots, n\}$, $A=\left\{a_{1}, a_{2}, \cdots, a_{i}, \cdots, a_{m}\right\}$ be a finite alternative set, where $a_{i}$ denotes the $i^{\text {th }}$ alternative, and $C=\left\{c_{1}, c_{2}, \cdots, c_{j}, \cdots, c_{n}\right\}$ be a finite criteria set, where $c_{j}$ denotes the $j^{\text {th }}$ criterion. Let $W=\left(w_{1}, w_{2}, \cdots, w_{j}, \cdots, w_{n}\right)^{T}$ be the criterion weight vector, where $w_{j}$ denotes the weight or degree of importance of criterion $c_{j}$, and $\sum_{j=1}^{n} w_{j}=1,0 \leq w_{j} \leq 1$, and $j=1,2, \cdots, n$. In addition, let $S=\left\{s_{i} \mid i=1,2, \cdots, 2 t-1, t \in N\right\}$ be the linguistic term set, and let $X=\left[x_{i j}\right]_{m \times n}$ be a decision matrix, where $x_{i j}$ is the linguistic evaluation of alternative $a_{i}$ with respect to criterion $c_{j}$. Furthermore, let $x_{i j} \in S, i \in M$, and $j \in N$.

Since different decision-makers have different reference points, defining a fixed reference point for every decision-maker would be unreasonable. Thus, the reference point selection principles provided in Section 3 are used in the proposed approach to obtain an appropriate reference point for each decision-maker under every criterion. 
In the remainder of this section, cloud model and prospect theory are used to develop an FMCDM approach with the predetermined reference point selection principles. The proposed approach is comprised of the following steps.

Step 1: Select a reference point for each decisionmaker.

A reference point is selected for each decision-maker based on the criteria values of the linguistic terms. Let $S=\left\{s_{i} \mid i=1,2, \cdots, 2 t-1, t \in N\right\} \quad$ be a pre-established finite and totally ordered linguistic term set with odd cardinality, where $s_{i}$ represents the $i^{\text {th }}$ linguistic variable of set $S$, and $t$ represents the cardinality of the set ${ }^{23}$.

According to the reference point selection principles described in Section 3, for a decision-maker with prior purchasing experiences, his or her reference point $Y_{j}^{R}$ to an item under the $j^{\text {th }}$ criterion can be determined using Algorithm 1; otherwise, the reference point can be determined using Algorithm 2.

Step 2: Transform the linguistic terms into clouds.

Linguistic evaluations can be aggregated and transformed into clouds, which are described by three digital characteristics, by utilizing the BCGs. For example, the linguistic evaluations $X=\left[x_{i j}\right]_{m \times n}$ of an item $a_{i}$ under criterion $c_{j}$ can be aggregated and transformed into the cloud evaluation $Y_{i j}\left(E x_{i j}, E n_{i j}, H e_{i j}\right)$, where $E x_{i j}$ is the expected value of $X, E n_{i j}$ is the entropy of $E x_{i j}$, and $H e_{i j}$ is the hyper entropy. These three digital characteristics reflect the overall evaluations of item $a_{i}$ under criterion $c_{j}$.

Step 3: Calculate the cloud prospect values of each item under each criterion.

The cloud prospect value $v_{i j}$ of the $i^{\text {th }}$ item under the $j^{\text {th }}$ criterion can be calculated using Equation (6) provided in Definition 5. First, the cloud evaluation $Y_{i j}$ and the decision-maker's reference point $Y_{j}^{R}$ are compared using Definition 3. Next, the distance between $Y_{i j}$ and $Y_{j}^{R}$, expressed as $d\left(Y_{i j}, Y_{j}^{R}\right)$, can be calculated using Definitions 2. The value of $d\left(Y_{i j}, Y_{j}^{R}\right)$ reflects the deviation of an item value from the reference point. Then, $\pi\left(p_{i j}\right)$, which reflects the decision-maker's risk attitude to risk, can also be calculated using Equation (6). Finally, the cloud prospect value $v_{i j}$ of the $i^{\text {th }}$ item under the $j^{\text {th }}$ criterion can be calculated using Equation (6).
Step 4: Calculate the comprehensive cloud prospect value of each item.

$$
V_{i}=\sum_{j=1}^{n} w_{j} v_{i j}, i=1,2, \cdots, m
$$

The comprehensive cloud prospect value of an item can be calculated using Equation (7), in which $w_{j}$ denotes the weight of the $j^{\text {th }}$ criterion, $v_{i j}$ denotes the cloud prospect value of the $i^{\text {th }}$ item under the $j^{\text {th }}$ criterion, and $V_{i}$ denotes the comprehensive cloud prospect value of the $i^{\text {th }}$ item.

Step 5: Rank the items.

The items can be ranked according to their comprehensive cloud prospect values $V_{i}$. Higher values of $V_{i}$ are associated with higher-satisfaction items.

\section{Illustrative example and comparative analysis}

In this section, a purchasing decision problem is used to demonstrate the applicability of the proposed FMCDM approach based on cloud model and prospect theory. In addition, a comparative study and discussion is performed in order to verify the applicability and accuracy of the proposed approach.

\subsection{Illustrative example}

As mentioned previously, consumer reviews can significantly influence the purchasing decisions of potential customers. However, as a consequence of the large number of evaluations available to potential customers, selecting alternatives and making effective purchasing decisions can be difficult. Consumers who purchase a single lens reflex (SLR) camera may choose to provide an evaluation of that item on an e-commerce website, and a potential customer who is considering purchasing an SLR camera may make a decision based on these evaluations.

Taobao.com, a network retail business platform, was founded by Alibaba group on May 10, 2003. Taobao.com has become the largest $\mathrm{C} 2 \mathrm{C}$ website in China, with more than 60 million visits each day. Currently, there are nearly 5 billion users and more than 8 billion items available for purchase on this website. Approximately 48 thousand items are sold on Taobao.com per minute. Although the same item can be listed for sale by multiple sellers, the quality of that item can differ significantly depending on its source. Thus, ecommerce can be risky for the customer. In order to 
combat this riskiness, potential customers oftentimes rely heavily on information provided by other consumers.

In the following example, linguistic evaluations of SLR cameras provided by a potential customer are collected in order to determine his reference point. Linguistic evaluations of alternative SLR cameras provided by other consumers are also collected in order to further assist the customer with his or her purchasing decision.

First, a potential customer was asked to select a few SLR cameras from Taobao.com. Then, the characteristics of these SLR cameras were analyzed using the evaluations provided by other customers in order to determine which SLR cameras might be preferred by the potential customer. The terms $a_{1}, a_{2}, a_{3}$, and $a_{4}$ were used to denote the alternative SLR camera options. Three criteria were considered during the analysis, including battery performance $\left(c_{1}\right)$, screen performance $\left(c_{2}\right)$, and image quality $\left(c_{3}\right)$. The weight vector of the three criteria was given as $w=(0.3,0.25$, $0.45)^{\mathrm{T}}$. The linguistic term evaluations were obtained from Taobao.com.
The purchasing-making decision procedure based on cloud model and prospect theory was comprised of the following steps.

Step 1: Determine the reference point of the potential customer.

On Taobao.com, item evaluations are provided in linguistic terms with a linguistic term set of

$$
\begin{aligned}
S=\left\{s_{1}\right. & =\text { VP:very poor }, s_{2}=\text { P:poor, } s_{3}=\text { M:medium, } \\
& \left.s_{4}=\text { H:high, } s_{5}=\text { VH:very high }\right\} .
\end{aligned}
$$

As shown in Table 4, the customer in this example purchased and provided linguistic evaluations for 20 SLR cameras.As shown in Table 5, the reference points for the SLR camera under each criterion were obtained using Algorithm 1.

Table 5 Reference points for the SLR camera under each criterion

\begin{tabular}{cccc}
\hline Item & $c_{1}$ & $c_{2}$ & $c_{3}$ \\
\hline SLR camera & $(3.8,0.78,0.95)$ & $(3.95,0.77,0.96)$ & $(3.85,0.61,0.75)$ \\
\hline
\end{tabular}

Step 2: Transform the linguistic terms into clouds.

The linguistic evaluations of the alternative items $a_{1}$, $a_{2}, a_{3}$, and $a_{4}$ are shown in Tables 6-9, respectively.

Table 4 Linguistic evaluations of the SLR camera under each criterion

\begin{tabular}{llll}
\hline Items & $c_{1}$ & $c_{2}$ & $c_{3}$ \\
\hline Canon 100D & $V H$ & $V H$ & $V H$ \\
Canon 1000D & $H$ & $M$ & $M$ \\
Nikon D5100 & $P$ & $H$ & $H$ \\
Canon 350D & $M$ & $V H$ & $H$ \\
Nikon D300S & $M$ & $M$ & $V H$ \\
Canon 600D & $M$ & $V H$ & $H$ \\
Nikon D800 & $H$ & $H$ & $M$ \\
Canon 700D & $V H$ & $M$ & $H$ \\
Canon 10D & $H$ & $V H$ & $V H$ \\
Nikon D7100 & $M$ & $H$ & $M$ \\
Canon 550D & $P$ & $M$ & $H$ \\
Nikon D5200 & $V H$ & $V H$ & $H$ \\
Canon 650D & $H$ & $H$ & $V H$ \\
Nikon D2HS & $V H$ & $M$ & $M$ \\
Canon 20Da & $H$ & $V H$ & $H$ \\
Nikon D100 & $H$ & $M$ & $M$ \\
Canon 7D2 & $H$ & $H$ & $M$ \\
Canon 400D & $H$ & $V H$ & $M$ \\
Nikon D40X & $M$ & $P$ & $H$ \\
Nikon D3000 & $V H$ & $H$ & $H$ \\
\hline & & & \\
& $H$ & $H$ \\
\hline
\end{tabular}

Table 6 Linguistic evaluations of $a_{1}$

\begin{tabular}{ccc}
\hline$c_{1}$ & $c_{2}$ & $c_{3}$ \\
\hline$H$ & $V H$ & $H$ \\
$H$ & $H$ & $V H$ \\
$M$ & $H$ & $V H$ \\
$V H$ & $H$ & $H$ \\
$M$ & $V H$ & $H$ \\
$H$ & $M$ & $M$ \\
$V H$ & $H$ & $H$ \\
$H$ & $M$ & $H$ \\
$M$ & $M$ & $V H$ \\
$M$ & $V H$ & $M$ \\
$V H$ & $V H$ & $V H$ \\
$H$ & $H$ & $H$ \\
$V H$ & $H$ & $H$ \\
$M$ & $M$ & $M$ \\
$H$ & $V H$ & $H$ \\
$V H$ & $H$ & $H$ \\
$H$ & $M$ & $M$ \\
$M$ & $H$ & $H$ \\
$H$ & $V H$ & $H$ \\
$H$ & $M$ & $H$ \\
\hline & &
\end{tabular}


Table 7 Linguistic evaluations of $a_{2}$

\begin{tabular}{ccc}
\hline$c_{1}$ & $c_{2}$ & $c_{3}$ \\
\hline$M$ & $H$ & $H$ \\
$M$ & $H$ & $H$ \\
$H$ & $M$ & $V H$ \\
$H$ & $H$ & $M$ \\
$M$ & $M$ & $V H$ \\
$V H$ & $M$ & $V H$ \\
$H$ & $M$ & $M$ \\
$M$ & $H$ & $M$ \\
$H$ & $H$ & $M$ \\
$H$ & $M$ & $V H$ \\
$M$ & $H$ & $M$ \\
$M$ & $M$ & $V H$ \\
$H$ & $M$ & $H$ \\
$H$ & $M$ & $H$ \\
$H$ & $H$ & $M$ \\
$V H$ & $H$ & $V H$ \\
$M$ & $H$ & $M$ \\
$M$ & $H$ & $V H$ \\
$M$ & $V H$ & $M$ \\
$M$ & $M$ & $V H$ \\
\hline & &
\end{tabular}

Table 8 Linguistic evaluations of $a_{3}$

\begin{tabular}{ccc}
\hline$c_{1}$ & $c_{2}$ & $c_{3}$ \\
\hline$V H$ & $V H$ & $M$ \\
$V H$ & $H$ & $V H$ \\
$V H$ & $M$ & $H$ \\
$H$ & $M$ & $H$ \\
$M$ & $M$ & $H$ \\
$M$ & $H$ & $M$ \\
$M$ & $V H$ & $M$ \\
$V H$ & $M$ & $M$ \\
$M$ & $M$ & $M$ \\
$V H$ & $M$ & $M$ \\
$V H$ & $M$ & $M$ \\
$M$ & $H$ & $M$ \\
$M$ & $M$ & $V H$ \\
$M$ & $M$ & $V H$ \\
$M$ & $M$ & $M$ \\
$M$ & $V H$ & $M$ \\
$M$ & $M$ & $M$ \\
$M$ & $V H$ & $V H$ \\
$M$ & $H$ & $H$ \\
$M$ & $H$ & $M$ \\
\hline & &
\end{tabular}

Table 9 Linguistic evaluations of $a_{4}$

\begin{tabular}{ccc}
\hline$c_{1}$ & $c_{2}$ & $c_{3}$ \\
\hline$H$ & $H$ & $M$ \\
$H$ & $H$ & $V H$ \\
$V H$ & $M$ & $M$ \\
$M$ & $M$ & $M$ \\
$V H$ & $M$ & $V H$ \\
$V H$ & $H$ & $M$ \\
$M$ & $H$ & $M$ \\
$M$ & $V H$ & $V H$ \\
$M$ & $V H$ & $V H$ \\
$V H$ & $M$ & $M$ \\
$M$ & $M$ & $M$ \\
$V H$ & $V H$ & $M$ \\
$H$ & $M$ & $V H$ \\
$H$ & $V H$ & $V H$ \\
$M$ & $V H$ & $M$ \\
$V H$ & $V H$ & $V H$ \\
$M$ & $M$ & $V H$ \\
$V H$ & $M$ & $M$ \\
$M$ & $V H$ & $V H$ \\
$V H$ & $V H$ & $V H$ \\
\hline & &
\end{tabular}

The linguistic evaluations of each alternative SLR camera under the three criteria were transformed into aggregated cloud values using BCGs. The results are shown in Table 10.

Table 10 Aggregated cloud evaluations of the alternative SLR cameras

\begin{tabular}{clll}
\hline Items & \multicolumn{1}{c}{$c_{1}$} & \multicolumn{1}{c}{$c_{2}$} & \multicolumn{1}{c}{$c_{3}$} \\
\hline$a_{1}$ & $(4,0.67,0.75)$ & $(4,0.67,0.75)$ & $(4,0.58,0.50)$ \\
$a_{2}$ & $(3.65,0.45,0.65)$ & $(3.60 .52,0.75)$ & $(3.6,0.45,0.68)$ \\
$a_{3}$ & $(3.65,0.71,1.06)$ & $(3.6,0.68,0.95)$ & $(3.6,0.63,0.90)$ \\
$a_{4}$ & $(4,0.7,1.00)$ & $(4,0.71,1.00)$ & $(4,0.73,1.25)$ \\
\hline
\end{tabular}

Step 3: Calculate the cloud prospect value of each item under each criterion.

The cloud prospect value of each alternative SLR camera under each criterion was calculated using Equation (6). In this process, $p_{\mathrm{i}}$ was identified as the positive review rate of the $i^{\text {th }}$ item and we adopted $\alpha=\beta=0.88, \lambda=2.25, \gamma=0.61$ and $\delta=0.72$ of Equation (6) based on the results of numerous experiments conducted by Kahneman and Tversky ${ }^{46,62}$. The calculation results are shown in Table 11. 
Table 11 Cloud prospect values of the alternatives under each criterion

\begin{tabular}{cccc}
\hline Items & $c_{1}$ & $c_{2}$ & $c_{3}$ \\
\hline$a_{1}$ & 0.08371 & 0.06921 & 0.08502 \\
$a_{2}$ & 0.11397 & -0.232 & -0.16351 \\
$a_{3}$ & -0.0866 & -0.16953 & -0.12871 \\
$a_{4}$ & 0.05784 & 0.02572 & -2.38372 \\
\hline
\end{tabular}

Step 4: Calculate the comprehensive cloud prospect value of each item.

The comprehensive cloud prospect value of each alternative SLR camera was obtained using Equation (7) as follows:

$V_{1}=0.0807, V_{2}=-0.0974, V_{3}=-0.1263, V_{4}=-0.1039$.

Step 5: Rank the items.

Since the cloud prospect values of the SLR cameras were ranked as $V_{1}>V_{2}>V_{4}>V_{3}$, the rankings of the items can be obtained as $a_{1} \succ a_{2} \succ a_{4} \succ a_{3}$, the customer would be most satisfied with option $a_{1}$.

\subsection{Comparative study and discussion}

In order to verify the applicability of the proposed FMCDM approach, a comparative study was performed using two other commonly used decision-making approaches that utilize linguistic information. The SLR camera example presented in Subsection 5.1 was used to conduct the comparative analysis. The ranking results are shown in Table 12.

Table 12 Ranking results obtained by the three approaches

\begin{tabular}{cccc}
\hline Approaches & The final ranking & $\begin{array}{c}\text { The best } \\
\text { item(s) }\end{array}$ & $\begin{array}{c}\text { The } \\
\text { worst } \\
\text { item(s) }\end{array}$ \\
\hline $\begin{array}{c}\text { The approach } \\
\text { developed in Ref. } \\
\begin{array}{c}\text { 50 } \\
\text { The approach } \\
\text { developed in Ref. }\end{array}\end{array}$ & $a_{4} \succ a_{1} \succ a_{3} \succ a_{2}$ & $a_{4}$ & $a_{2}$ \\
$\begin{array}{c}\text { The proposed } \\
\text { approach }\end{array}$ & $a_{1} \succ a_{2} \succ a_{4} \succ a_{2} \succ a_{3}$ & $a_{1}$ & $a_{3}$ \\
& & $a_{1}$ & $a_{3}$ \\
\hline
\end{tabular}

The proposed approach was compared to two other approaches. First of all, the approach was compared to the approach developed in Ref. ${ }^{50}$. In that approach, the linguistic terms were transformed into clouds using the golden section method and aggregated using cloud aggregation operators based on expected utility theory.
The alternatives were then ranked according to their final aggregated values. According to the results, the proposed method and the method developed in Ref. ${ }^{50}$ yielded different final rankings. These results were attributed to two differences in these methods. The approach developed in Ref. ${ }^{50}$ is based on expected utility theory, conventional arithmetic weighted average operators are used as cloud aggregation operators. Thus, items with more positive linguistic evaluations are ranked higher than other items. However, based on prospect theory, reference points and loss aversion are considered during the decision-making process in the proposed approach. Hence, a consumer may select an item that may not have the highest aggregated value if it satisfies his or her subjective expectations and risk preferences. In addition, in the approach developed in Ref. ${ }^{50}$, clouds are generated via golden section. Generally, items with a larger number of $s_{2}$ or $s_{4}$ evaluations may be assigned lower orders because the Ex of the cloud is smaller than it should be according to gold section method. However, in the proposed approach, clouds are generated with BCGs, so it avoids the problems associated with the golden section method.

Next, the proposed approach was also compared to the approach developed by Liu in Ref. ${ }^{63}$. In the approach developed by Liu, prospect theory was utilized to handle decision-making problems under risk with uncertain linguistic information, and the probability is defined as the interval value. According to Liu's approach, the linguistic evaluations were transformed into uncertain linguistic variables, and the statistical probability of each item receiving rave reviews was determined. Using the resulting information, the prospect values of the alternative items were calculated, and the items were ranked, as shown in Table 12. Also, the proposed method and the method developed in Ref. ${ }^{63}$ yielded identical maximum and minimum rankings. However, items $a_{2}$ and $a_{4}$ received different rankings. These rankings were not the same due to the differences in the reference point selection principles of the two approaches. In Liu's approach, a fixed reference point, calculated as the median of the linguistic variables, is employed. In our approach, novel reference point selection principles are utilized in order to determine the reference point of that particular customer.

Unlike the approaches developed in Ref. ${ }^{50}$ and Ref. ${ }^{63}$, the subjective expectations and risk preferences of a customer, as well as the specific reference point for that 
particular customer, are considered in the proposed approach. The final item rankings obtained via the proposed approach, $a_{1} \succ a_{2} \succ a_{4} \succ a_{3}$, were more precise and reliable than the rankings obtained via the other methods. Thus, the proposed purchasing decision approach could be effectively used by consumers in order to maximize customer satisfaction and minimize risk.

\section{Conclusion}

In this paper, an FMCDM purchasing decision-making approach that takes into account the customer's subjective expectations and aversion to loss in an ecommerce environment is developed. In the proposed approach, linguistic evaluations are transformed into clouds, and prospect theory and reference point selection principles are used to calculate the cloud prospect values of alternative items. The advantage of the approach is that it considers the unique subjective expectation and loss aversion of a customer in real-life purchasing decision-making process in e-commerce environment. Combining cloud model and prospect theory, the proposed approach could be applied to some scenarios with unique potential customers. Thus, the rankings obtained via the proposed approach reflect the preferences of customers more properly than the rankings obtained via other approaches.

Further studies concerning the proposed approach should be conducted. First of all, the proposed approach only considers linguistic evaluations, limiting the consumer's ability to express his or her feelings regarding an item. These linguistic evaluations could be transformed into fuzzy linguistic sets, such as intuitionistic fuzzy sets and neutrosophic sets, in order to maximize the potential of FMCDM approaches. In addition, the criteria weights used for the purposes of this paper were provided directly by a consumer in the illustrative example. However, in an actual e-commerce environment, asking customers to provide criteria weights would be unpractical. Thus, a method to purchasing problems employing objectively calculating weights should be developed. Moreover, the similarity between decision-makers needs to be carefully studied, because it will affect the final rankings of alternatives when using Algorithm 2. Some similarity measurements should be discussed and selected to find similar decision-makers, and the number and weights of similar decision-makers should also be discussed and identified.
Besides, further studies can also focus on improving the effectiveness of the proposed approach.

\section{Conflict of Interests}

The authors declare that there is no conflict of interests regarding the publication of this paper.

\section{Acknowledgement}

This work was supported by the National Natural Science Foundation of China (Nos. 71501192 and 71210003). The authors also would like to express appreciation to the anonymous reviewers and editors for their very helpful comments that improved the paper.

\section{References}

1. R.E. Deacon and F.M. Firebaugh, Mary winter instructor's manual to accompany family resource management: Principles and applications, Boston: Allyn and Bacon, 1981.

2. A.D. Prawitz, M.L. Cantwell, F.C. Lawrence, and L.R. Welch, A strategy for teaching consumer decision-making, Journal of Consumer Education, 9 (1991) 26-33.

3. F.M. Nicosia, Consumer decision processes: Marketing and advertising implications, New Jersey: Prentice-Hall Incorporated, 1966.

4. J.F. Engel, D.T. Kollat, and R.D. Blackwell, Consumer behavior (4th ed), New York: Holt, Rinchart \& Winston, 1982.

5. C.P. Puto, The framing of buying decisions, Journal of Consumer Research, 14 (1987) 301-315.

6. H. Mintzberg, D. Raisinghani, and A. Théorêt, The structure of "unstructured" decision processes, Administrative Science Quarterly, 21 (1976) 246-275.

7. S. Shim, M.A. Eastlick, S.L. Lotz, and P. Warrington, An online prepurchase intentions model: The role of intention to search, Journal of Retailing, 77 (2001) 397-416.

8. T. Zhang and D. Zhang, Agent-based simulation of consumer purchase decision-making and the decoy effect, Journal of Business Research, 60 (2007) 912-922.

9. D.J. Kim, D.L. Ferrin, and H.R. Rao, A trust-based consumer decision-making model in electronic commerce: The role of trust, perceived risk, and their antecedents, Decision Support Systems, 44 (2008) 544-564.

10. C. Carlsson and R. Fullér, Fuzzy multiple criteria decision making: Recent developments, Fuzzy Sets and Systems, 78 (1996) 139-153.

11. A. Özgen, G. Tuzkaya, U.R. Tuzkaya, and D. Özgen, A multi-criteria decision making approach for machine tool selection problem in a fuzzy environment, International Journal of Computational Intelligence Systems, 4 (2011) 431-445.

12. C. Kahraman, S.C. Onar, and B. Oztaysi, Fuzzy multicriteria decision-making: A literature review, International Journal of Computational Intelligence Systems, 8 (2015) 637-666. 
13. M. Afshin, S. Reza, and M. Najmeh, Multi-criteria group purchasing decision-making process based on marketing mix (4Ps) using fuzzy TOPSIS, Journal of American Science, 9 (2012) 309-313.

14. Ö. Tosun and G. Akyüz, A fuzzy TODIM approach for the supplier selection problem, International Journal of Computational Intelligence Systems, 8 (2015) 317-329.

15. Z.P. Tian, J. Wang, J.Q. Wang, and X.H. Chen, Multicriteria decision-making approach based on gray linguistic weighted Bonferroni mean operator, International Transactions in Operational Research, (2015) DOI: 10.1111/itor. 12220 .

16. Z.P. Tian, J. Wang, J.Q. Wang, and H.Y. Zhang, Simplified neutrosophic linguistic multi-criteria group decisionmaking approach to green product development, Group Decision and Negotiation, (2016) DOI: 10.1007/s10726016-9479-5.

17. H.Y. Zhang, P. Ji, J.Q. Wang, and X.H. Chen, An improved weighted correlation coefficient based on integrated weight for interval neutrosophic sets and its application in multi-criteria decision-making problems, International Journal of Computational Intelligence Systems, 8 (2015) 1027-1043.

18. Z.P. Tian, H.Y. Zhang, J.Q. Wang, and X.H. Chen, Multicriteria decision-making method based on a cross-entropy with interval neutrosophic sets, Journal of Systems Science, (2015) DOI: 10.1080/00207721.2015.1102359.

19. H.Y. Zhang, J.Q. Wang, and X.H. Chen, An outranking approach for multi-criteria decision-making problems with interval-valued neutrosophic sets, Neural Computing and Applications, 27 (2016) 615-627.

20. J.H. Hu, K.L. Xiao, X.H. Chen, and Y.M. Liu, Interval type-2 hesitant fuzzy set and its application in multicriteria decision making, Computers \& Industrial Engineering, 87 (2015) 91-103.

21. W. Duan, B. Gu, and A.B. Whinston, Do online reviews matter? An empirical investigation of panel data, Decision Support Systems, 45 (2008) 1007-1016.

22. I. Titov and R. McDonald, Modeling online reviews with multi-grain topic models, in: Proceedings of The 17th International Conference on World Wide Web, $A C M$, 2008, pp. 111-120.

23. F. Herrera, E. Herrera-Viedma, and J.L. Verdegay, A model of consensus in group decision making under linguistic assessments, Fuzzy Sets and Systems, 78 (1996) 73-87.

24. M. Delgado, J.L. Verdegay, and M.A. Vila, Linguistic decision-making models, International Journal of Intelligent Systems, 7 (1992) 479-492.

25. Y.P. Jiang, Z.P. Fan, and J. Ma, A method for group decision making with multi-granularity linguistic assessment information, Information Sciences, 178 (2008) 1098-1109.

26. Z. Zhang and C. Guo, A method for multi-granularity uncertain linguistic group decision making with incomplete weight information, Knowledge-Based Systems, 26 (2012) 111-119.

27. Z. Xu, Uncertain linguistic aggregation operators based approach to multiple attribute group decision making under uncertain linguistic environment, Information Sciences, 168 (2004) 171-184.

28. Z. Xu, Induced uncertain linguistic OWA operators applied to group decision making, Information Fusion, 7 (2006) 231-238.

29. Y.X. Ma, J.Q. Wang, J. Wang, and X.H. Wu, An interval neutrosophic linguistic multi-criteria group decisionmaking method and its application in selecting medical treatment options, Neural Computing and Applications, (2016) DOI: 10.1007/s00521-016-2203-1.

30. L. Martínez and F. Herrera, An overview on the 2-tuple linguistic model for computing with words in decision making: Extensions, applications and challenges, Information Sciences, 207 (2012) 1-18.

31. L. Martínez, D. Ruan, and F. Herrera, Computing with words in decision support systems: An overview on models and applications, International Journal of Computational Intelligence Systems, 3 (2010) 382-395.

32. R. Rosa M and M. Luis, An analysis of symbolic linguistic computing models in decision making, International Journal of General Systems, 42 (2013) 121-136.

33. J. Wang, J.Q. Wang, H.Y. Zhang, and X.H. Chen, Multicriteria group decision-making approach based on 2-tuple linguistic aggregation operators with multi-hesitant fuzzy linguistic information, International Journal of Fuzzy Systems, 18 (2016) 81-97.

34. J.Q. Wang, D.D. Wang, H.Y. Zhang, and X.H. Chen, Multi-criteria group decision making method based on interval 2-tuple linguistic information and Choquet integral aggregation operators, Soft Computing, 19 (2015) 389-405.

35. J.Q. Wang, P. Wang, H.Y. Zhang, and X.H. Chen, Atanassov's interval-valued intuitionistic linguistic multicriteria group decision-making method based on trapezium cloud model, IEEE Transactions on Fuzzy Systems, 6 (2015) 542-554.

36. H.Y. Zhang, P. Ji, J.Q. Wang, and X.H. Chen, A neutrosophic normal cloud and its application in decisionmaking, Cognitive Computation, (2016) DOI:10.1007/ s12559-016-9394-8.

37. Z.P. Tian, J. Wang, J.Q. Wang, and H.Y. Zhang, A likelihood-based qualitative flexible approach with hesitant fuzzy linguistic information, Cognitive Computation, (2016) DOI: 10.1007/s12559-016-9400-1.

38. D.Y. Li and Y. Du, Artificial intelligence with uncertainty, London: Chapman \& Hall/CRC, 2007.

39. D.Y. Li, J.W. Han, X.M. Shi, and M.C. Chan, Knowledge representation and discovery based on linguistic atoms, Knowledge-Based Systems, 10 (1998) 431-440.

40. W. Zhang, S. Liu, B. Sun, Y. Liu, and M. Pecht, A cloud model-based method for the analysis of accelerated life test data, Microelectronics Reliability, 55 (2015) 123-128.

41. Z.Y. Xu, Z.L. Wu, J. Yao, and Y.Q. Ren, Vessel traffic system project post evaluation method based on cloud model, Applied Mechanics and Materials, 687-691 (2014) 1528-1531.

42. T. Wu, J. Xiao, K. Qin, and Y. Chen, Cloud model-based method for range-constrained thresholding, Computers \& Electrical Engineering, 42 (2015) 33-48.

43. H.L. Wang and Y.Q. Feng, On multiple attribute group decision making with linguistic assessment information based on cloud model, Control and Decision, 20 (2005) 679-685.

44. K. Zhao, J.W. Gao, Z.Q. Qi, and C.B. Li, Multi-criteria 
risky-decision-making approach based on prospect theory and cloud model, Control and Decision, 30 (2015) 395402.

45. C. Barnard and H. Simon, Administrative behavior: A study of decision-making processes in administrative organization, New York: Free Press, 1947.

46. D. Kahneman and A. Tversky, Prospect theory: An analysis of decision under risk, Econometrica, 47 (1979) 263-291.

47. F. Meng, C. Tan, and X. Chen, An approach to Atanassov's interval-valued intuitionistic fuzzy multi-attribute decision making based on prospect theory, International Journal of Computational Intelligence Systems, 8 (2015) 591-605.

48. P. Liu, F. Jin, X. Zhang, Y. Su, and M. Wang, Research on the multi-attribute decision-making under risk with interval probability based on prospect theory and the uncertain linguistic variables, Knowledge-Based Systems, 24 (2011) 554-561.

49. J. Ren, Linguistic-stochastic multi-criterion decisionmaking method based on cloud model, Computer Integrated Manufacturing Systems, 18 (2012) 2792-2797.

50. J.Q. Wang, L. Peng, H.Y. Zhang, and X.H. Chen, Method of multi-criteria group decision-making based on cloud aggregation operators with linguistic information, Information Sciences, 274 (2014) 177-191.

51. K.C. Di, D.Y. Li, and D.R. Li, Cloud theory and its applications in spatial data mining and knowledge discovery, Journal of Image and Graphics, 4 (1999) 930935.

52. D.Y. Li, H.J. Meng, and X.M. Shi, Membership clouds and membership cloud generators, Journal of Computer Research and Development, 32 (1995) 15-20.

53. C.Y. Liu, D.Y. Li, Y. Du, and X. Han, Some statistical analysis of the normal cloud model, Information and Control, 34 (2005) 236-239.
54. G.W. Zhang, D.Y. Li, P. Li, J.C. Kang, and G.S. Chen, A collaborative filtering recommendation algorithm based on cloud model, Journal of Software, 18 (2007) 24032411.

55. R.A. Jarvis and E.A. Patrick, Clustering using a similarity measure based on shared near neighbors, IEEE Transactions on Computers, C-22 (1973) 1025-1034.

56. X. Liu, Entropy, distance measure and similarity measure of fuzzy sets and their relations, Fuzzy Sets and Systems, 52 (1992) 305-318.

57. T.R. dos Santos and L.E. Zárate, Categorical data clustering: What similarity measure to recommend?, Expert Systems with Applications, 42 (2015) 1247-1260.

58. H. Ma and Z. Hu, Recommend trustworthy services using interval numbers of four parameters via cloud model for potential users, Frontiers of Computer Science, 9 (2015) 887-903.

59. J.B. Si, Y. Yao, W.Y. Guo, and F. Yang, Web user cluster method and realization based on fuzzy clustering, Journal of Jilin University, 43 (2013) 485-488.

60. Y.X. Wang, J. Xu, Y.M. Zhang, and G. Xiao, Customer cluster analysis method and application based on customized product, Journal of Zhejiang University of Technology, 37 (2009) 406-410.

61. P.S. Hiremath, S.P. Algur, and S. Shivashankar, Cluster analysis of customer reviews extracted from web pages, Journal of Applied Computer Science \& Mathematics, 4 (2010) 56-62.

62. A. Tversky and D. Kahneman, Advances in prospect theory: Cumulative representation of uncertainty, Journal of Risk and Uncertainty, 5 (1992) 297-323.

63. P.D. Liu, Method for multi-attribute decision-making under risk with the uncertain linguistic variables based on prospect theory, Control and Decision, 26 (2011) 893-897. 